\title{
O Monitoramento Metacognitivo em Tarefas que Envolvem a Criatividade Verbal
}

\author{
Luma Tiziotto Deffendi \\ Patrícia Waltz Schelini \\ Universidade Federal de São Carlos
}

\begin{abstract}
RESUMO - A metacognição é entendida como o conhecimento que o indivíduo possui sobre seus próprios processos cognitivos e que permite o monitoramento, a regulação e a avaliação de suas atividades cognitivas, inclusive das atividades envolvendo criatividade. O objetivo deste estudo foi investigar o monitoramento metacognitivo de universitários em tarefas envolvendo processos criativos verbais. Participaram da pesquisa 82 universitários, sendo a eles apresentado o Teste de Pensamento Criativo de Torrance - Versão Verbal. Após a aplicação do teste de criatividade, eles foram solicitados a emitir estimativas sobre o seu desempenho, que correspondem ao monitoramento metacognitivo. Os resultados sugerem que os participantes parecem possuir poucas habilidades de monitoramento metacognitivo nas tarefas propostas, visto que as correlações entre os desempenhos reais e estimados foram fracas.
\end{abstract}

Palavras-chave: processos cognitivos, metacognição, monitoramento, criatividade, universitários

\section{Metacognition Monitoring in Tasks that Involve Verbal Creativity}

\begin{abstract}
Metacognition involves the knowledge that individuals have with respect to their own cognitive processes, and includes self- monitoring, self-regulation and evaluation of their cognitive activities, including activities evolving creativity. The aim of this study was to investigate metacognitive monitoring among university students when performing tasks that involve verbal creative processes. Participants included 82 university students, who completed the Torrance's Verbal Test of Creative Thinking. After finishing the creativity test, they were asked to evaluate their performance (metacognitive monitoring). The results suggest that the participants appear to have poor metacognitive monitoring skills in tasks involving creativity, since the correlation between actual and estimated performance was weak.
\end{abstract}

Keywords: cognitive processes, metacognition, monitoring, creativity, college students

Pioneiro ao referir-se à metacognição, Flavell (1970) definiu-a como o conhecimento que o sujeito tem sobre o seu próprio conhecimento. $\mathrm{O}$ termo significa, essencialmente, a cognição da cognição, ou seja, o conhecimento dos próprios processos e produtos cognitivos, ou algo relacionado com eles (Flavell, 1976; Flavell \& Wellman, 1977).

As hipóteses de Flavell expandiram o conceito de metacognição do simples conhecimento sobre a própria cognição para a concepção de que esse conhecimento pode ser utilizado pelo indivíduo para planejar, monitorar, regular e avaliar suas atividades cognitivas, como o raciocínio, a compreensão, a solução de problemas e a aprendizagem (Jou \& Sperb, 2006; Woolfolk, 2000), com a sugestão de implicações práticas do conceito. Em 1979, Flavell apresentou o Modelo de Monitoramento Cognitivo, sugerindo que o monitoramento cognitivo pode ser entendido pela interação de quatro classes de fenômenos: conhecimento metacognitivo, experiências metacognitivas, objetivos e ações ou estratégias.

O conhecimento metacognitivo é definido como o conhecimento ou crença que o sujeito possui sobre si próprio, sobre os fatores da pessoa, da tarefa e da estratégia bem como sobre o modo como tais variáveis afetam o resultado dos procedimentos cognitivos. Contribui para o controle das condutas de resolução, permitindo ao sujeito reconhecer e

1 Endereço para correspondência: Rua Flauzino Marques, 10, Apto. 43, Jd. Alvorada, São Carlos, SP, Brasil. CEP: 13.562-002. E-mail: lumadeffendi@yahoo.com.br representar as situações, ter mais fácil acesso ao repertório das estratégias disponíveis e selecionar as suscetíveis de se poderem aplicar. Permite, também, avaliar os resultados finais e/ou intermédios e reforçar a estratégia escolhida ou alterá-la, em função das avaliações feitas (Ribeiro, 2003).

As experiências metacognitivas, por sua vez, são experiências conscientes, cognitivas e afetivas (Flavell, 1981). A sua principal função é a de informar o sujeito sobre o ponto em que ele se encontra na atividade, as suas principais dificuldades e o tipo de progresso que ele já fez ou que tem a possibilidade de fazer.

O terceiro aspecto do modelo diz respeito aos objetivos, implícitos ou explícitos, que impulsionam e mantêm o empreendimento cognitivo. Tais objetivos podem ser impostos por alguém ou selecionados pelo próprio indivíduo, o qual pode modificar o seu objetivo no decorrer da realização da tarefa, no caso de discordar da imposição feita a ele (Ribeiro, 2003).

Finalmente, a quarta classe, que são as estratégias, referem-se às cognições ou a outros comportamentos que conduzem ao progresso ou à avaliação dos progressos cognitivos. Elas têm, por sua vez, a finalidade de conduzir ao desenvolvimento cognitivo e podem surgir na sequência da ação das primeiras quando, diante de uma avaliação da situação, conclui-se pela necessidade da utilização de novas estratégias (Flavell, 1987).

A unificação da teoria sobre metacognição coube a Nelson e Narens $(1990,1996)$, que deram um importante 
passo quando propuseram um modelo para explicar o fluxo de informação no sistema metacognitivo. De acordo com o modelo, o sistema metacognitivo é formado pelo nível meta, de atuação metacognitiva, e pelo nível objeto, que seria o nível de atuação das capacidades e dos processos cognitivos como atenção, percepção, memória, linguagem, aprendizagem e resolução de problemas (Nelson \& Narens, 1990, 1996). Na teoria de Nelson e Narens, dois conceitos são fundamentais: monitoramento e controle metacognitivos. O monitoramento corresponde à capacidade de observar, refletir e experienciar $\mathrm{o}$ andamento dos processos cognitivos e, com isso, julgar ou caracterizar o funcionamento cognitivo (Nelson \& Narens, 1996). Ocorre quando a informação flui do nível objeto para o nível meta, sendo que o monitoramento do nível objeto se dá por meio da construção de um modelo, entendido como a representação mental da realidade do momento cognitivo. Assim, esse tipo de fluxo de informação se caracteriza pelo acesso ou avaliação do progresso, ou estado atual, de uma determinada atividade cognitiva. De acordo com Dunlosky e Metcalfe (2009), para investigar o monitoramento metacognitivo é comum solicitar que os participantes julguem um estado cognitivo. $\mathrm{O}$ monitoramento, portanto, pode ser útil na identificação de problemas que instigam a modificação de planos ou estratégias (Desoete, 2008) e permite que o indivíduo estime algo sobre o seu desempenho cognitivo, bem como sobre quão ajustado esse desempenho está em relação à demanda apresentada pelas tarefas.

O produto do monitoramento metacognitivo é chamado de julgamento (Efklides, 2006; Nelson \& Narens, 1994; Son \& Schwartz, 2002). Segundo Pieschl (2009), existem duas dimensões relevantes dos julgamentos: o seu momento de emissão e a sua abrangência. Os indivíduos formulam julgamentos em três momentos distintos: antes, durante ou após a realização de uma tarefa. Julgamentos feitos antes da realização de uma tarefa (prospectivos) correspondem às expectativas sobre a realização dessa tarefa. Em relação à abrangência, os julgamentos podem se referir a um item específico ou a um conjunto de itens que compõem uma tarefa. Além disso, para que o julgamento do indivíduo seja uma ferramenta útil na avaliação do funcionamento metacognitivo, é necessário compará-lo com o desempenho de fato apresentado por ele.

Os fatores que interferem no julgamento apontados por Maki e McGuire (2002), dificuldade e familiaridade com o tema, podem ser classificados de acordo com os critérios apresentados por Flavell (1979): a forma de apresentação da tarefa, a dificuldade do texto e a divisão do texto em trechos podem ser agrupados na categoria das variáveis de tarefa, enquanto a familiaridade com o tema (conteúdo da tarefa) e a experiência com as situações de avaliação podem ser consideradas variáveis da pessoa. Segundo os autores, níveis intermediários de dificuldade geram julgamentos mais acurados do que níveis fáceis ou muito difíceis, e o domínio sobre o tema favorece a compreensão e precisão dos julgamentos (Maki \& McGuire, 2002).

As tarefas podem ser classificadas de acordo com o grau de complexidade, ou seja, o nível de conhecimento e operações cognitivas exigidas por cada atividade. Atividades de recordação de informações têm menor grau de complexidade do que tarefas que exijam compreensão de conteúdos; estas, por sua vez, são menos complexas do que tarefas que exijam aplicação do conhecimento adquirido. Este seria mais um exemplo de propriedade-estado, que influencia o julgamento (Pieschl, 2009).

A tendência atual, segundo Son e Schwartz (2002), é que as pesquisas da área abordem as tentativas de compreender a relação entre monitoramento e controle metacognitivos. Ou seja, os pesquisadores buscam compreender as estratégias utilizadas pelos indivíduos e como elas podem ser aprimoradas, tanto na codificação (aprendizagem) quanto na recordação de informações (Zampieri, 2012).

A metacognição envolve planejamento, monitoramento e regulação não só dos processos cognitivos, mas também dos afetivos e comportamentais (Boruchovitch \& Santos, 2006). O pensar sobre o próprio pensar, ou pensamento de segunda ordem, permitiu ao ser humano observar e corrigir seus pensamentos e suas ações, desenvolvendo estratégias cada vez mais acuradas para interagir com o meio e, portanto, para garantir sua sobrevivência (Jou \& Sperb, 2006).

\section{A Metacognição na Criatividade}

Na medida em que o ser humano é um sujeito metacognitivo por natureza, detentor de um sistema de "autocorreção" para saber o que aprendeu e o que deve aprender, pode-se supor que o conhecimento metacognitivo esteja de certa maneira relacionado com a criatividade na sua dimensão mais cognitiva. Refletir, planejar, organizar estratégias, usar o conhecimento previamente adquirido, atentar-se a informações relevantes, tudo isso leva a um processo criativo (Almeida \& Seminério, 1998). Na expressão da criatividade, não só interessa o ato de pensar, mas também a autorreflexão dos indivíduos sobre a maneira como o fazem e como o poderiam fazer "melhor", ou seja, a sua avaliação metacognitiva do processo criativo.

Em 1956 e depois em 1967, Guilford trabalhou em sua teoria fatorial da inteligência segundo a qual operações cognitivas (memória, pensamento convergente, pensamento divergente, avaliação cognição) aplicadas aos diferentes tipos de informações resultariam em diferentes tipos de produções. Baseado nas provas de pensamento divergente de Guilford, Torrance (1966) vai igualmente se interessar pela criatividade. $\mathrm{O}$ autor fez, inicialmente em seus trabalhos, a distinção entre quatro medidas de pensamento criativo (fluência, flexibilidade, elaboração e originalidade) e, com base nesses parâmetros, avaliou o desempenho dos sujeitos nos seus testes (Torrance \& Safter, 1999). Entretanto, a partir de 1980, esse autor passou a demonstrar insatisfação com a limitação dos conceitos utilizados para avaliação da criatividade, uma vez que acabavam por reduzir a medida do construto à medida do pensamento divergente.

A fim de demonstrar a validade preditiva de seus testes e de comprovar que eles poderiam avaliar de maneira precisa a criatividade dos indivíduos, Torrance $(1972,1981,1992)$ realizou um importante estudo longitudinal em que provou a correspondência entre vários indicadores da criatividade figurativa presentes nos testes e a produção na vida real dos indivíduos. Com base nos resultados obtidos, o autor 
adicionou novos indicadores da criatividade aos seus testes bem como incorporou novas categorias de análise e de correção, e eles passaram a avaliar, além dos aspectos cognitivos da criatividade, também os aspectos afetivos ou emocionais (Wechsler, 2004).

O desenvolvimento de estudos de validação de tais instrumentos para a população brasileira foi realizado por Wechsler (2002b), e suas pesquisas demonstraram a adequação cultural desses instrumentos para o Brasil ao confirmarem a validade discriminativa dos testes verbal e figural como um forte indicador de criatividade. Embora a pesquisa internacional ofereça dados que indicam a possibilidade de identificação transcultural dos traços e habilidades da pessoa criativa, sabe-se que existem características próprias de cada população que só tem sentido se compreendidos em seu contexto histórico e cultural.

São necessários vários tipos de interações para que a criatividade seja expressa de modo harmônico para o indivíduo e também para a sociedade. Wechsler (2005) salienta, em sua concepção, três grandes conjuntos que interagem. O primeiro deles é composto pelas habilidades cognitivas; e o segundo, pela área afetiva. Da intercessão desses dois conjuntos surge o terceiro: os estilos de criar, que podem ser entendidos como a intersecção das habilidades cognitivas e as características da pessoa criativa, facilitando assim o processo criativo. O desenvolvimento desse processo dependerá também da influência do ambiente, que pode ser escolar, familiar, profissional e/ou da sociedade (Wechsler, 2005).

Muitos pesquisadores, além do interesse em conceituar criatividade, têm voltado a sua atenção às características das pessoas criativas. A linha de investigação que destaca a "pessoa criativa" centra-se no estudo das características pessoais que facilitam ou entravam a produção dos atos criativos, nomeadamente, os valores, as atitudes, as motivações (Sternberg \& Lubart, 1996), as habilidades cognitivas, os estilos de aprendizagem, os estilos de criatividade (Alencar, Fleith, \& Bruno-Faria, 2010) e, ainda, os interesses, os níveis de autonomia, autoconfiança, perseverança, espontaneidade, sensibilidade emocional e de tolerância à frustração (Pereira, 1996).

Wechsler (2002a) é uma das autoras que melhor explorou as habilidades por trás das características das pessoas criativas. Por meio de estudos empíricos de análise de biografias e de critérios comparativos, ela enfatizou as seguintes características: (a) a fluência, que diz respeito à capacidade de gerar um grande número de soluções ou ideias diante de uma situação específica; (b) a flexibilidade, entendida como a mudança de perspectiva ao se olhar um problema; (c) o pensamento original e inovador que quebra os padrões habituais de pensar (é a capacidade de produzir ideias raras e incomuns); (d) a alta sensibilidade externa e interna, que se caracteriza pela percepção de falhas nas informações dadas ou adquiridas e a percepção de sentimentos de desconforto interno; (e) a fantasia e a imaginação, que é uma brincadeira interiorizada que pode ser utilizada na resolução de problemas e conflitos; (f) o inconformismo, independência de julgamentos e abertura a novas experiências, que possibilitam acreditar nas próprias ideias para a produção criativa, a despeito dos outros; (g) o uso de analogias e combinações incomuns, que pode ser descrito como brincar com ideias, cores, formas e conceitos a fim de se conseguir justaposições improváveis; (h) as ideias elaboradas e enriquecidas que significam o detalhamento das formas finais da ideia, ou seja, a transformação dessa ideia em produto.

As características e habilidades das pessoas criativas poderiam ser mais bem aproveitadas e trabalhadas no ensino superior, em vista de aumentar as chances de os jovens recém-formados obterem êxito no mercado de trabalho. Um dos caminhos para tornar a universidade mais criativa é desenvolver as habilidades criativas nos estudantes, professores e direção. Para isso, torna-se necessária a investigação acerca do entrelaçamento das variáveis envolvidas no fenômeno criativo. Se o potencial dessas características fosse valorizado e utilizado em favor da educação, seria mais fácil ensinar os estudantes a pensar de modo criativo, bem como a conhecer e saber explorar da melhor forma as suas capacidades cognitivas.

\section{Objetivos}

O estudo teve como objetivo geral investigar o monitoramento metacognitivo de estudantes universitários por meio do julgamento (estimativa) sobre o desempenho em tarefas que envolvem processos criativos verbais. Os objetivos específicos foram (a) analisar as relações entre o real desempenho em uma medida de criatividade verbal e o desempenho estimado (julgamento metacognitivo) pelos participantes considerando a mesma medida e (b) investigar as relações entre o real desempenho nas características criativas de fluência, flexibilidade, elaboração e originalidade bem como as estimativas de desempenho (julgamentos) referentes a essas características

\section{Método}

\section{Participantes e Local}

Participaram do estudo 82 estudantes universitários cursando entre primeiro e nono semestre de graduação, sendo 34 do gênero masculino e 48 do gênero feminino, de sete cursos diferentes de duas universidades públicas, localizadas em um município do interior do estado de São Paulo. A média de idade da amostra foi de 22,5 anos e, entre os participantes, havia 49 estudantes de Psicologia, 24 de Música, 2 de Engenharia Elétrica, 2 de Matemática, 3 de Bacharelado em Sistemas da Informação, 1 de Engenharia da Computação e 1 de Bacharelado em Ciência da Computação. A coleta de dados foi realizada em salas disponibilizadas pela instituição de ensino frequentada por eles, com cadeiras e mesas suficientes para acomodá-los, livre de ruídos ou outras atividades que pudessem comprometer a condução do procedimento. A seleção dos participantes se deu de dois modos: contato via e-mail com professores de algumas disciplinas (já conhecidos das pesquisadoras) e contato direto 
com alguns alunos das próprias universidades (amostra de conveniência).

\section{Materiais}

Teste de Pensamento Criativo de Torrance - Versão Verbal. A criatividade foi avaliada por meio do Teste de Pensamento Criativo de Torrance - Versão verbal, adaptado por Wechsler (2004) e composto por seis atividades, para as quais são solicitadas perguntas, causas, consequências ou ideias para melhoria de produtos. As respostas dos participantes foram avaliadas de acordo com características ou indicadores cognitivos e emocionais relacionados com a criatividade, identificados nas pesquisas relacionadas com a pessoa criativa, em estudos internacionais e nacionais (Torrance \& Safter, 1999; Wechsler, 2004).

Entre as 10 características criativas avaliadas pelo teste de criatividade verbal estão: (a) fluência, (b) flexibilidade, (c) elaboração, (d) originalidade, (e) expressão da emoção, (f) fantasia, (g) perspectiva incomum e (h) uso de analogias e metáforas. A análise das respostas ao Teste de Criatividade Verbal também permite a obtenção do índice criativo verbal 1 (ICV1) e do índice criativo verbal 2 (ICV2). O primeiro é baseado nos escores para o conjunto das características fluência, flexibilidade, originalidade e elaboração. Essas quatro características são consideradas como indicadoras de pensamento divergente. Já o ICV2 é a soma dos escores para o conjunto das oito características criativas verbais, somando as quatro características presentes no ICV1 com o restante das características consideradas os aspectos afetivos da personalidade criativa.

Técnica de Monitoramento da Criatividade. Para avaliar a metacognição dos participantes foi utilizada a Técnica de Monitoramento da Criatividade - TMC (Deffendi \& Schelini, 2015), destinada à verificação dos julgamentos (estimativas) dos participantes em relação aos próprios desempenhos no teste de criatividade. Para isso, os participantes estimaram, após a realização do teste de Torrance e de acordo com cada uma das quatro características do ICV1, se consideraram ter conseguido: (a) apresentar ideias importantes, (b) apresentar diversas ideias, (c) apresentar ideias detalhadas, (d) apresentar ideias incomuns e (e) propor soluções criativas, de modo geral, para cada uma das atividades do teste. A TMC é uma escala numérica, que varia de 0 a 10 e é composta por seis itens relativos a cada uma das atividades do teste mais um item final sobre o desempenho no teste de modo geral.

Os itens da técnica foram criados a partir da descrição das características criativas que compõem o ICV1 (fluência, flexibilidade, elaboração e originalidade) apresentadas por Wechsler (2004) no manual do teste de Avaliação da Criatividade por Palavras - Versão Verbal. A técnica foi construída de modo que o participante possa julgar o seu desempenho de acordo com a sua capacidade de gerar um grande número de ideias diante da tarefa (fluência); mudar de perspectiva ao olhar o problema (flexibilidade); detalhar as ideias propostas para solucionar o problema; propor soluções inovadoras para o problema, quebrando com os padrões habituais de pensamento (originalidade); e propor soluções criativas de modo geral. Tais características foram escolhidas entre todas pois são as que se referem aos aspectos cognitivos da criatividade.

\section{Procedimento}

A participação dos estudantes ocorreu condicionalmente ao seu interesse e consentimento. O Termo de Consentimento Livre e Esclarecido, contendo informações sobre os procedimentos e objetivos da pesquisa, foi entregue antes da aplicação dos instrumentos. Só foram submetidos ao procedimento aqueles que estavam de acordo com esse documento e com a sua participação. Após os participantes receberem o Termo de Consentimento Livre e Esclarecido e concordarem com a participação na pesquisa, eles passaram por uma única sessão experimental coletiva de aproximadamente 50 minutos, em que responderam, por escrito e em um caderno de resposta individual, ao Teste de Pensamento Criativo de Torrance - Versão Verbal e à Técnica de Monitoramento da Criatividade. Vale elucidar que a aplicação do procedimento proposto foi aprovada pelo Comitê de Ética (número do processo: 17478113.9.0000.5504).

\section{Resultados}

No que se refere ao ICV1, as respostas dos 82 participantes apresentaram uma distribuição que tendeu à normalidade (Média $=93,59$; Desvio Padrão $=38,52$ ). Por essa razão, serão utilizados testes estatísticos paramétricos para realizar as análises posteriores (correlacionais) que incluirão a medida de criatividade na amostra total.

A Tabela 1 mostra as correlações de Pearson entre as pontuações totais no teste de criatividade e na TMC, em que ICV1 corresponde à pontuação total para esse índice e MetGeral à pontuação no último item da escala, que avalia a estimativa do participante sobre o seu desempenho como um todo no teste de criatividade. Já Flutotal, Fletotal, Elatotal e Origtotal correspondem, respectivamente, às somatórias das estimativas da fluência, flexibilidade, elaboração e originalidade atribuídas pelos participantes em cada uma das seis atividades do Teste de Pensamento Criativo de Torrance - Versão Verbal. A Al geral corresponde à pontuação total do primeiro item da técnica, bem como A2 geral à pontuação total do segundo item, e assim sucessivamente.

Considerando as correlações estabelecidas entre o ICV1 e as pontuações obtidas na TMC, é possível constatar que houve correlações significativas, porém fracas, entre esse índice e as estimativas de fluência $(\mathrm{r}=0,235)$, flexibilidade $(\mathrm{r}=0,331)$ e originalidade $(\mathrm{r}=0,263)$, proporcionadas pela escala que avalia a metacognição.

Uma correlação fraca $(r=0,19)$ e não significativa foi encontrada entre o ICV1 e a Metacognição Geral (MetGeral), ou seja, entre os escores obtidos no teste de criatividade (considerando-se as quatro características que compõem o ICV1) e a estimativa geral sobre esse desempenho feita na TMC. Para elaboração, a correlação também foi fraca ( $\mathrm{r}$ $=-0,100)$, não significativa e negativa. 
Tabela 1: Correlações de Pearson entre o Índice Criativo Verbal 1 e diferentes pontuações obtidas na Técnica de Monitoramento da Criatividade

\begin{tabular}{|c|c|c|c|c|c|c|c|c|c|c|c|c|c|}
\hline & ICV1 & MetGeral & Flutotal & Fletotal & Elatotal & Origtotal & A1geral & A2geral & A3geral & A4geral & A5geral & A6geral & \\
\hline ICV1 & & & 0,191 & $0,235^{*}$ & $0,331 * *$ & $-0,100$ & $0,263^{*}$ & 0,160 & 0,196 & 0,169 & 0,066 & 0,027 & 0,124 \\
\hline MetGeral & & & & $0,745^{* *}$ & $0,735^{* *}$ & $0,479 * *$ & $0,729 * *$ & $0,744 * *$ & $0,609 * *$ & $0,691 * *$ & $0,587 * *$ & $0,690 * *$ & $0,410 * *$ \\
\hline Flutotal & & & & & $0,810^{* *}$ & $0,473 * *$ & $0,717^{* *}$ & $0,644 * *$ & $0,629 * *$ & $0,591 * *$ & $0,667 * *$ & $0,690^{* *}$ & $0,344 * *$ \\
\hline Fletotal & & & & & & $0,572 * *$ & $0,764 * *$ & $0,658^{* *}$ & $0,642 * *$ & $0,679 * *$ & $0,596^{* *}$ & $0,622 * *$ & $0,342 * *$ \\
\hline Elatotal & & & & & & & $0,504 * *$ & $0,485^{* *}$ & $0,489 * *$ & $0,497 * *$ & $0,366^{* *}$ & $0,370^{* *}$ & $0,249 *$ \\
\hline Origtotal & & & & & & & & $0,766^{* *}$ & $0,758^{* *}$ & $0,744^{* *}$ & $0,654 * *$ & $0,660^{* *}$ & $0,382 * *$ \\
\hline
\end{tabular}

Nota. ${ }^{*}$ Correlação de Pearson significativa a $0,05 . * *$ Correlação de Pearson significativa a 0,01 .

Considerando-se o ICV1 e os seis itens da técnica, individualmente (em que $\mathrm{A} 1$ geral corresponde à pontuação total do primeiro item da escala, bem como A2 geral à pontuação total do segundo item, e assim sucessivamente), obteve-se correlações positivas, muito fracas e não significativas.

A Tabela 2 traz os dados referentes às correlações entre as pontuações individuais de cada um dos índices que compõem o ICV1 - ou seja, fluência, flexibilidade, elaboração e originalidade - e as estimativas de desempenho referentes a estes índices na TMC. Considerando as correlações entre as pontuações indicativas do desempenho no Teste de Pensamento Criativo de Torrance - Versão Verbal (desempenho real) e os desempenhos estimados na TMC, encontraram-se correlações significativas e positivas entre fluência no teste de criatividade (Tflu) e fluência estimada na técnica que avalia a metacognição (Flutotal; $r=0,252$ ), entre flexibilidade no teste de criatividade (TFlex) e flexibilidade estimada na técnica que avalia a metacognição (Fletotal; $\mathrm{r}=0,255$ ), entre elaboração no teste de criatividade (Telab) e elaboração estimada na técnica que avalia a metacognição (Elatotal; $r=0,208$ ) e, finalmente, entre originalidade no teste de criatividade (Torig) e originalidade estimada na técnica que avalia a metacognição (Origtotal; $r=0,249$ ). Contudo, tais correlações foram fracas.

\section{Discussão}

A fim de produzir conhecimento a respeito do monitoramento metacognitivo, a coleta, a descrição e a discussão dos resultados do presente estudo tiveram como objetivo principal investigar o monitoramento metacognitivo de estudantes universitários por meio do julgamento (estimativa) sobre o desempenho em tarefas que envolveram processos criativos verbais. Analisar as relações entre o real desempenho em uma medida de criatividade verbal e o desempenho estimado (julgamento metacognitivo) pelos participantes considerando a mesma medida e investigar as relações entre o real desempenho nas características criativas de fluência, flexibilidade, elaboração e originalidade e as estimativas de desempenho (julgamentos) referentes a essas características foram objetivos específicos do presente estudo.

De modo geral, obtiveram-se correlações fracas entre o real desempenho no teste de criatividade e o desempenho estimado (julgamentos) pelos participantes por meio da TMC. As pontuações no ICV1 e na Metacognição Geral (MetGeral) não se correlacionaram significativamente, de modo que não houve relação entre o desempenho real no teste de criatividade e a estimativa sobre esse desempenho. Isso pode sugerir uma dificuldade por parte dos participantes em monitorar metacognitivamente o seu desempenho em uma tarefa que envolva criatividade. Além disso, as correlações entre a pontuação real no teste de criatividade, considerandose o ICV1, e cada uma das dimensões da técnica que avalia a metacognição (estimativas de fluência, flexibilidade, elaboração e originalidade) também foram fracas e não significativas.

Uma hipótese para explicar as baixas correlações encontradas é que os participantes podem ter uma autoobservação deficitária quanto aos processos relativos à criatividade envolvidos no Teste de Pensamento Criativo de Torrance - Versão Verbal. Se for o caso, isso explicaria por que as estimativas de desempenho não tenderam a estabelecer relações fortes e significativas com o desempenho global no teste de criatividade (considerando-se o ICV1), ainda

Tabela 2: Correlações de Pearson entre os índices de Fluência, Flexibilidade, Elaboração e Originalidade e as estimativas de desempenho referentes a estes índices na Técnica de Monitoramento da Criatividade

\begin{tabular}{ccccccccc}
\hline & TFlex & TElab & TOrig & MetGeral & Flutotal & Fletotal & Elatotal & Origtotal \\
\hline TFlu & $0,792^{* *}$ & 0,090 & $0,776^{* *}$ & 0,178 & $0,252^{*}$ & $0,344^{* *}$ & $-0,150$ & $0,249^{*}$ \\
TFlex & & 0,118 & $0,777^{* *}$ & 0,170 & 0,202 & $0,255^{*}$ & $-0,008$ & 0,177 \\
TElab & & & 0,167 & 0,067 & 0,006 & 0,134 & $0,208^{*}$ & 0,150 \\
TOrig & & & & 0,167 & 0,182 & $0,238^{*}$ & $-0,175$ & $0,249 *$ \\
\hline
\end{tabular}

Nota. ${ }^{*}$ Correlação de Pearson significativa a $0,05 .{ }^{* *}$ Correlação de Pearson significativa a 0,01 . As nomenclaturas TFlu, TFlex, Telab e TOrig referem-se, respectivamente, às pontuações totais (desempenho real) em Fluência, Flexibilidade, Elaboração e Originalidade no teste de criatividade. Já as nomenclaturas Flutotal, Fletotal, Elatotal e Oritotal representam as pontuações totais (desempenho estimado) feitas na Técnica de Monitoramento da Criatividade (TMC) para Fluência, Flexibilidade, Elaboração e Originalidade. 
mais se considerada a técnica de avaliação da metacognição retrospectiva utilizada no estudo. Segundo Desoete (2008), na técnica retrospectiva, ou seja, na formulação de julgamentos depois da realização da tarefa, indivíduos com baixa capacidade de auto-observação podem optar por respostas que não descrevam com muita fidedignidade seu desempenho.

Ainda observando as correlações estabelecidas entre o real desempenho no teste de criatividade e as pontuações obtidas na TMC, é possível constatar que houve correlações positivas e significativas, porém fracas, entre o ICV1 e as estimativas para fluência, flexibilidade e originalidade. Novamente, os dados parecem sugerir uma dificuldade no monitoramento metacognitivo, uma vez que a relação entre pontuação estimada e pontuação real no teste de criatividade foi fraca, chegando a não ser significativa, sendo inclusive negativa entre o ICV1 e a estimativa de desempenho em elaboração.

Outra hipótese que explicaria as baixas correlações entre real desempenho no teste de criatividade e desempenho estimado na técnica que avalia o monitoramento metacognitivo é a complexidade das atividades propostas pelo Teste de Pensamento Criativo de Torrance - Versão Verbal. Pieschl (2009) argumenta que as tarefas podem ser classificadas de acordo com o grau de complexidade, ou seja, o nível de conhecimento e operações cognitivas exigidas por cada atividade. Atividades de recordação de informações têm menor grau de complexidade do que tarefas que exijam compreensão de conteúdos; estas, por sua vez, são menos complexas do que tarefas que exijam aplicação do conhecimento adquirido. As atividades do teste podem ser consideradas complexas na medida em que, para se alcançarem soluções fluentes, flexíveis, elaboradas e originais, muitas operações cognitivas são exigidas. Além disso, as instruções que orientam os participantes para a resolução dos problemas propostos pelo teste explicitam que não há respostas certas ou erradas, ampliando a gama de possibilidades possíveis para essa resolução e dificultando a posterior formulação de estimativas acerca do real desempenho.

No que se refere às correlações entre os índices de fluência, flexibilidade, elaboração e originalidade e as estimativas de desempenho referentes a esses índices na TMC, os resultados apontaram para a existência de correlações positivas, fracas e significativas entre as estimativas de desempenho (julgamentos para fluência, flexibilidade, elaboração e originalidade, individualmente) e o real desempenho no teste de criatividade em cada um desses índices. A fraca relação entre os julgamentos (estimativas) e o desempenho real fortalecem a hipótese da dificuldade de monitoramento metacognitivo durante a realização das atividades propostas pelo teste de criatividade, considerando cada uma das dimensões avaliadas pelo teste.

Outra análise que deve ser feita em relação às baixas correlações encontradas é sobre a eficácia da TMC em avaliar o monitoramento metacognitivo dos participantes nas tarefas propostas pelo teste de criatividade. Levanta-se a hipótese de que talvez a técnica não tenha representado tão adequadamente as noções cognitivas de criatividade representadas pelo ICV1, avaliadas pelo Teste de Pensamento
Criativo de Torrance - Versão Verbal. Como já mencionado anteriormente nesta seção, pode-se supor que a maneira com que as características de fluência, flexibilidade, elaboração e originalidade foram apresentadas aos participantes, por meio das afirmativas presentes na escala, tenha gerado diversas interpretações dos conceitos e, consequentemente, confusão sobre o que realmente estaria sendo avaliado, dificultando as estimativas de desempenho.

Embora não seja novidade que o reconhecimento de que a educação superior é um terreno fértil para o desenvolvimento da criatividade (Chambers, 1973; Stewart, 1964; Yamamoto, 1975), o processo de ensino-aprendizagem, tal como adotado geralmente nessas instituições, dificulta o processo criativo dos alunos (Freeman, 2006). Em geral, a acurácia dos julgamentos tende a aumentar, conforme o indivíduo adquire maior familiaridade com a tarefa que executa (Efklides, 2006; Schraw, 2009). Logo, as baixas relações podem ser explicadas sob a ótica da pouca exposição dos universitários às propostas criativas, uma vez que a baixa exposição pode dificultar o treino de monitorar a execução de tarefas que envolvam a criatividade.

Além disso, o monitoramento metacognitivo em tarefas que envolvam a criatividade pode ser mais difícil porque, nesse tipo de tarefa, parece não haver respostas certas ou erradas como em tarefas cognitivas que envolvam respostas mais objetivas, com maior possibilidade para a constatação do acerto ou erro na emissão das respostas. A familiaridade com a tarefa é uma das variáveis apontadas como facilitadora da emissão de julgamentos acurados (Maki \& McGuire, 2002). Conforme explicam Zampieri e Schelini (2013a; 2013b), tarefas que não compõem o currículo escolar típico, ou seja, que tendem a não ser ensinadas ou treinadas diretamente, representam maior dificuldade para a formulação de julgamentos acurados sobre esse desempenho. Isso ocorre porque, para essas tarefas, há, geralmente, ausência de devolutivas e correções para informar o indivíduo acerca de seu desempenho.

Partindo-se do pressuposto que os feedbacks sobre a criatividade não são comuns como os referentes a outras tarefas cognitivas que requerem respostas objetivas, como as que envolvem matemática, leitura e escrita, por exemplo, justifica-se a dificuldade dos participantes para monitorar metacognitivamente o seu desempenho em tarefas que envolvem criatividade. Conforme explicam Maki e McGuire (2002), é possível considerar que o contato com informações a respeito do desempenho ofereça condições para que o indivíduo compare suas experiências metacognitivas e estimativas com seu desempenho real.

\section{Considerações Finais}

Este estudo proporcionou a constatação de que os universitários parecem possuir poucas habilidades de monitoramento metacognitivo em tarefas que envolvam a criatividade, visto que as relações entre os desempenhos reais e estimados tenderam a ser fracas. É necessário que as investigações acerca da temática continuem, no sentido de levantar novas hipóteses e de eliminar possíveis vieses 
proporcionados pelas limitações metodológicas encontradas no decorrer da aplicação do procedimento proposto.

Pode-se apontar como limitação a homogeneidade da amostra, que não contemplou cursos de graduação variados, muito embora tenha sido representativa para os cursos de Psicologia e Música, que corresponderam a aproximadamente $89 \%$ da amostra. Sugere-se, para próximas investigações, uma maior heterogeneidade para a variável curso dos participantes, de modo que a influência da mesma possa ser considerada na análise posterior dos resultados.

Outro aspecto que deve ser considerado em futuras investigações é a aplicabilidade da TMC, que precisa ser aprimorada para acessar mais adequadamente as noções de criatividade avaliadas pelo Teste de Pensamento Criativo de Torrance - Versal Verbal. Conforme abordado na seção de discussão, a técnica pode ter apresentado conceitos amplos na descrição das características que compõem o ICV1 (fluência, flexibilidade, elaboração e originalidade), e isso pode ter relação com as baixas correlações encontradas entre real desempenho no teste de criatividade e desempenho estimado na escala.

Além disso, a criatividade figural não foi avaliada neste estudo, e é interessante que ela seja considerada em outra oportunidade a fim de que o monitoramento metacognitivo da criatividade aborde os componentes cognitivos dessa capacidade de modo global, e não apenas em sua dimensão verbal. Para uma compreensão ainda melhor sobre a metacognição, sugere-se, ainda, que o aspecto do conhecimento metacognitivo, e suas implicações para o monitoramento, também seja abordado em pesquisas futuras, tendo em vista que o conhecimento metacognitivo colabora na tomada de decisão consciente do indivíduo, ajudando-o a identificar e representar as situações, facilitando o acesso às estratégias disponíveis e a escolha das que podem ser aplicadas, além de permitir avaliar os resultados finais ou intermediários.

Outra contribuição desde estudo a ser destacada é a produção de dados ainda muito pouco explorados sobre a relação entre metacognição e a dimensão cognitiva da criatividade. A partir das informações já apresentadas de que o monitoramento metacognitivo adequado acompanha o bom desempenho cognitivo, torna-se interessante considerar a possibilidade de estimulação das habilidades metacognitivas como estratégia para otimização do desempenho dos indivíduos em tarefas com demanda cognitiva, incluindo-se a essa demanda as tarefas que envolvem criatividade.

Espera-se que o corpo de conhecimentos produzidos por este estudo seja um fator a motivar a continuação da investigação neste campo, a fim de que os estudos investigativos produzam como benefícios a formulação de novas hipóteses, bem como de programas de intervenção e estimulação de habilidades metacognitivas relacionadas à expressão da criatividade, em especial no ensino superior, que é um terreno fértil para o seu desenvolvimento.

\section{Referências}

Alencar, E. M. L. S., Fleith, D. S., \& Bruno-Faria, M. F. (2010). A medida da criatividade: Possibilidades e desafios. In E. M. L. S. Alencar, M. F. Bruno-Faria \& D. S. Fleith (Orgs.), Medidas de criatividade: Teoria e prática (pp.11-34). Porto Alegre: Artmed.

Almeida, M. F., \& Seminerio, F. L. P. (1998). O imaginário cognitivo: Uma fronteira entre consciência e inconsciente. Arquivos Brasileiros de Psicologia 49, 94-107.

Boruchovitch, E., \& Santos, A. A. A. (2006). Estratégias de aprendizagem: Conceituação e avaliação. Em A. P. Noronha, A. A. A. Santos \& F. F. Sisto (Orgs.), Facetas do fazer em avaliação psicológica (pp. 107-124). São Paulo: Ed.Vetor.

Chambers, J. A. (1973). College teachers: Their effect on creativity of students. Journal of Educational Psychology, 65(3), 326331.

Deffendi, L. T., \& Schelini, P. W. (2015). O monitoramento metacognitivo em tarefas que envolvem a criatividade verbal. Dissertação de Mestrado, Universidade Federal de São Carlos, São Carlos, SP, Brasil.

Desoete, A. (2008). Multi-method assessment of metacognitive skills in elementary school children: How you test is what you get. Metacognition Learning, 3(3) 189-206. doi: 10.1007/ s11409-008-9026-0

Dunslosky, J., \& Metcalfe, J. (2009). Metacognition. Los Angeles: Sage.

Efklides A. (2006). Metacognition and affect: What can metacognitive experiencies tell us about the learning process? Educational Research Review, 1(1), 3-14. doi:10.1016/j. edurev.2005.11.001

Flavell, J. H. (1970). Developmental studies of mediated memory. Em H. W. Reese \& L. P. Lipsitt (Eds.), Advances in child development and behavior (vol. 5). New York: Academic Press.

Flavell, J. H. (1976). Metacognitive aspects of problem solving. Em L. B. Resnik (Ed.), The Nature of Intelligence (pp. 231-235). Hillsdale: Lawrence Erlbaum.

Flavel, J. H. (1979). Metacognition and cognitive monitoring: A new area of cognitive developmental inquiry. American Psychologist, 34(10), 906-911.

Flavell, J. H. (1981). Metacognition and cognitive monitoring: A new area of cognitive-developmental inquiry. Em H. Parke (Orgs.), Contemporary readings in child psychology (pp. 165169). New York: McGraw Hill.

Flavell, J. H. (1987). Speculations about the nature and development of metacognition. Em F. E. Weinert \& R. H. Kluwe (Eds.), Metacognition, motivation and understanding (pp. 21-29). Hillsdale: Lawrence Erlbaum Associates.

Flavell, J. H., \& Wellman, H. M. (1977). Metamemory. Em R. V. Kail, \& O. W. Hagen (Eds.), Perspectives on the development of memory and cognition (pp. 3-33). Hillsdale, N.Y.: Lawrence Erlbaum Associates.

Freeman, J. (2006). Fostering creativity in university performance. Arts and Humanities in Higher Education, 5(1), 91-103.

Guilford, J. P. (1956). The structure of intellect. Psychological Bulletin, 53(4), 267-293.

Guilford, J. P. (1967). The nature of human intelligence. New York: McGrow-Hill.

Jou, G. I., \& Sperb, T. M. (2006). A metacognição como estratégia reguladora da aprendizagem. Psicologia: Reflexão e Crítica, 19(2), 177-185. 
Maki, R. H., \& McGuire, M .J. (2002). Metacognition for text: Findings and implications for education. Em T. J. Perfect \& B. L. Schwartz (Orgs), Applied Metacognition (pp. 39-67). Cambridge: University Press.

Nelson, T. O., \& Narens, L. (1990). Metamemory: A theoretical framework and new findings. Em G. H. Bower (Ed.), The psychology of learning and motivation (pp. 125-173). New York: Academic Press.

Nelson, T. O., \& Narens, L. (1994). Why investigate metacognition. Em J. Metcalfe \& A. P. Shimamura (Orgs.), Metacognition: Knowing about knowing (pp. 1-25). Cambridge, MA: MIT Press.

Nelson, T., \& Narens, L. (1996). Why investigate metacognition? Em J. Metclafe, A. P. Metcalfe \& A. P. Shimamura (Eds.), Metacognition: Knowing about knowing (pp. 1-27). Cambridge: MIT Press.

Pieschl, S. (2009). Metacognitive calibration: An extended conceptualization and potential applications. Metacognition Learning, 4(1), 3-31. DOI: 10.1007/s11409-008-9030-4

Ribeiro, C. (2003). Metacognição: Um apoio ao processo de aprendizagem. Psicologia: Reflexão e Crítica, 16(1), 109-116.

Schraw, G. (2009). A conceptual analysis of five measures of metacognitive monitoring. Metacognition Learning, 4(1), 33-45. doi: 10.1007/s11409-008-9031-3

Son, L. K., \& Schwartz, B. L. (2002). The relation between metacognitive monitoring and control. Em T. J. Perfect \& B. L. Schwartz (Orgs), Applied metacognition (pp.15-35). Cambridge: University Press.

Sternberg, R. J., \& Lubart, T. I. (1996). Investing in creativity. American Psychologist, 51(7), 677-688

Stewart, G. W. (1964). Higher education and creativeness. The Journal of Higher Education, 17, 32-39.

Torrance, E. P. (1966). Torrance tests of creative thinking. Lexington, MA: Personnel Press.

Torrance, E. P. (1972). Predictive validity of the Torrance Tests of Creative Thinking. Journal of Creative Behavior, 6, 232-236
Torrance, E. P. (1981). Empirical validation of criterion-reference indicators of creative ability through a longitudinal study. Creative Child and Adult Quarterly, 6,136-140.

Torrance, E. P. (1992). The beyonders in a thirty year longitudinal study of creative achievement. Roeper Review, 15, 131-134.

Torrance, E. P., \& Safter, H. T. (1999). Making the creative leap beyond. Buffalo, NY: Creative Education Foundation.

Wechsler, S. M. (2002a). Criatividade: Descobrindo e encorajando (3.ed.). São Paulo: Editora Livro Pleno.

Wechsler, S. M. (2002b). Avaliação da criatividade por figuras e palavras: Testes de Torrance versão brasileira. Campinas: Lamp / Impressão Digital do Brasil.

Wechsler, S. M. (2004). Avaliação da criatividade por figuras e palavras: Testes de Torrance - versão brasileira ( $2^{\mathrm{a}} \mathrm{ed}$. rev.). Campinas: IDB e LAMP / PUC-Campinas.

Wechsler, S. M. (2005). Avaliação da criatividade: Um enfoque multidimensional. Em S. M. Wechsler \& S. L. R. Guzzo (Orgs.), Avaliação psicológica: Perspectiva internacional (pp. 231-259). São Paulo: Casa do Psicólogo.

Woolfolk, A. (2000). Psicologia da educação (pp. 217-244). Porto Alegre: Artmed.

Yamamoto, K. (1975). Creativity and higher education: A review. Higher Education, 4(1975), 213-225.

Zampieri, M., \& Schelini, P. W. (2013a). Monitoramento metacognitivo de crianças de acordo com o nível de desempenho em medidas de capacidades intelectuais. Psico, 44(2), 280-287.

Zampieri, M., \& Schelini, P. W. (2013b).O Uso de Medidas Intelectuais na Análise do Monitoramento Metacognitivo de Crianças. Psicologia: Teoria e Pesquisa, 29(2), 81-88.

Zampieri, M. (2012). Investigação do monitoramento metacognitivo de crianças diante de medidas de capacidades intelectuais. Dissertação de mestrado, Universidade Federal de São Carlos, São Carlos, SP, Brasil. Disponível em: http://www. bdtd.ufscar.br/htdocs/tedeSimplificado//tde_busca/arquivo. php? $\operatorname{codArquivo}=5014$. 